\title{
Notas epistemológicas en torno a las investigaciones newtonianas sobre la luz y el color
}

Leonardo Ruiz

Universidad de Navarra ruizg.leonardo@gmail.com

La investigación más reciente en torno al célebre físico, matemático, filósofo y teólogo Isaac Newton ha tomado cada vez un marco más amplio de análisis. Se ha pasado del simple paradigma del gran hombre de ciencia a una concepción mucho más comprensiva y holística de su obra. En este sentido, una revisión del trabajo experimental y científico de Newton no basta para entender lo que subyace verdaderamente a su pensamiento; es necesario, entonces, hacer una cautelosa exploración de sus textos referidos a temas filosóficos e incluso teológicos ${ }^{1}$.

En el presente trabajo se pretende realizar un estudio acerca de las reflexiones newtonianas en torno a la luz y al color que revolucionaron la óptica de su tiempo y que, de algún modo, tienen raíces y consecuencias filosóficas y metodológicas que, de ser pasadas por alto, implicarían un costo demasiado alto. Para ello, será necesario realizar un análisis detallado del trabajo científico que desarrolló Newton en torno a estos

\footnotetext{
${ }^{1}$ Han sido ya varios los comentadores que siguen esta línea en la interpretación de Newton: ver Keynes 1963; Marquina 2006; Ducheyne 2001; McGuire 1980; y Benítez 2003.
} 
problemas; una vez hecho esto, podremos derivar de ahí los principios epistemológicos de su trabajo y la relación que en su pensamiento guardan la ciencia y la filosofía natural.

El texto que muestra de un modo más acabado la teoría newtoniana sobre la luz y el color es, desde luego, La Óptica o tratado de las reflexiones, refracciones, inflexiones y colores de la luz. Sin embargo, al igual que los Principia, la Óptica (dejadas de lado las Cuestiones finales) parece ser fruto de un esfuerzo por abocarse al mero análisis de ciertas propiedades de la materia y la luz sin profundizar en conceptos que vayan más allá de los fenómenos. No obstante, esta lectura de la Óptica — que llamaré cientificista ${ }^{2}$ - resulta poco útil para la interpretación del sistema newtoniano. En efecto, son estos conceptos tocantes a la filosofía natural (el «espacio y tiempo» absoluto, las «cantidades evanescentes», los «rayos de luz» en la Óptica) los que fundamentan todo su constructo científico, y por eso es inevitable que aparezcan, explícita o implícitamente, en estos textos.

Hay que decir, en favor de la lectura cientificista, que ciertamente es notorio el intento de Newton por utilizar los conceptos metafísicos en la forma más somera y utilizando únicamente los aspectos imprescindibles de ellos para justificar su sistema ${ }^{3}$. Sin embargo, esto no quiere decir que tales

\footnotetext{
${ }^{2}$ La discusión en torno al papel que juegan tales conceptos filosóficos o metafísicos en la ciencia newtoniana está, sin duda, en una constante controversia. Ya desde el siglo XIX, Ernst Mach (ver 1960) elaboró un agudo ataque contra estas nociones; podríamos decir que es, sin duda, uno de los grandes defensores de la lectura cientificista de Newton. Para una reconstrucción de las principales posturas que siguen esta lectura y sus posteriores polémicas, ver Strong 1952; y Toulmin 1959a y 1959b.

${ }^{3}$ Un claro ejemplo de esto son los conceptos de espacio y tiempo absolutos que en los Principia son descritos en un tono «funcionalista» para fundamentar todo el Sistema del Mundo newtoniano. Una perspectiva muy distinta a la que se presenta en el De gravitatione et aequipondio fluidorum donde hay una teorización sobre estos conceptos que va más allá de los simples fenómenos. El famoso Scholium Generale de los Principia, probablemente el fragmento más revelador sobre las ideas filosóficas de Newton en los textos científicos, fue
} 
conceptos no posean un alcance explicativo mayor al de sus aplicaciones prácticas dentro de los textos científicos; su uso en estos trabajos no los agota y por eso es necesario acudir a los textos no publicados y a las anotaciones de juventud.

Ha sido principalmente a partir de ciertas lecturas de los Principia y de la Óptica que se ha generado la imagen de Newton como un científico implacable que no da un paso más allá de la rigurosidad de los cálculos y los fenómenos; como dice Thomas Kuhn, es de estas monumentales creaciones que ha surgido la idea de Newton como «the Olympian father of modern science». Sin embargo, es importante notar que es precisamente en los textos de juventud y en sus cartas donde se observa — también en opinión de Kuhn- el creativo e innovador científico y filósofo que era (ver 1958; 29).

Es plausible, entonces, que exista una relación semejante entre el trabajo experimental de Newton y ciertos principios filosóficos en los temas relacionados con la luz y el color. El presente trabajo se abocará a mostrar cuál es la relación entre unos y otros en los textos newtonianos que poseen esta tópica. Para ello, haremos referencia a los primeros textos en donde Newton aborda esta temática (1); después, analizaremos un texto clave en esta materia, a saber, la Nueva teoría sobre la luz y el color (2 y 3); posteriormente haremos algunas reflexiones metodológicas sobre las conclusiones que Newton ahí propone ayudándonos con algunos textos posteriormente publicados, en concreto, analizaremos los problemas que la Nueva teoría plantea heurísticamente (4); el tema de

- como nos recuerda Brett- una inserción posterior por petición del editor de la segunda edición, Roger Cotes, para «eliminar algunos prejuicios que se han asentado copiosamente contra éste [Los Principia]». Las «Cuestiones de Óptica» fueron también incluidas por una recomendación de Samuel Clark, su editor y traductor (ver Strong 1952). 
la división cromática que hace Newton (5); y su posterior polémica con su contemporáneo Robert Hooke (6). Finalmente, daré mis conclusiones.

\section{Primeras aproximaciones de Newton a la diversa refractabilidad de los colores}

Las primeras investigaciones que registró Newton en torno a la naturaleza de los colores aparecen en Quaestiones Quaedam Philosophicae, el cuadernillo en donde Newton realizó apuntes variados durante su estancia en el Trinity College y en años posteriores. En el folio 36 de las Quaestiones, titulado «Of Colours», Newton adelanta por primera vez, como una anotación suelta, la alternativa a cuya solución dedicará varias décadas de su vida: "Colours arise either from shadows intermixed with light, or stronger \& weaker reflection or parts of the body mixed with \& carried away by light» $(1664 ; 36)^{4}$. En efecto, Newton está considerando la posibilidad de que la variación de color sea fruto de la combinación de distintas cantidades de negro y blanco. Ésta era, a grandes rasgos, la postura que sostenía Aristóteles y que se mantuvo en las controversias filosóficas, con mayor o menor éxito, hasta el Renacimiento (ver Guerlac 1986) ${ }^{5}$. Sin embargo, el mismo Newton parece descartarla enseguida pues, de ser así, los dibujos en blanco y negro deberían de verse coloreados a lo lejos, lo mismo que los bordes de las sombras (ver Newton 1664; 36).

\footnotetext{
${ }^{4}$ El segundo número se corresponde con la numeración que el mismo Newton realizó en su cuadernillo.

${ }^{5}$ Es altamente probable que Newton hubiera leído de primera mano los textos aristotélicos pues, como nos indica Escohotado (ver 1997; XIII), tras la coronación de Carlos II en 1660, se impone como obligatorio el tomismo en el Trinity College y, con ello, la lectura de Aristóteles.
} 
Varios folios después, en las Quaestiones, en el número 63 vuelve a aparecer el título «Of Colours». Esta vez se trata de un experimento realizado con un prisma triangular a través del cual se observa una pantalla bicolor. Newton realiza varias observaciones, cuando los colores que analiza en el cartón son el rojo y azul, se percata de un fenómeno especial:

That the rays which make blew are refracted more than the rays which make red appeares from this experiment If one hafe of the thred abc be blew \& the other red \& a shade or black body be put behind it then lookeing on the thred through a prism one halfe of the thred shall appeare higher than the other \& not both in one direct line, by reason of unequall refractions in the 2 differing colours $(1664 ; 36)$.

Newton se percata de que el azul y el rojo poseen distintos índices de refracción cuando son vistos a través de un prisma. Este experimento no es fortuito, sino que sigue una larga línea de investigación de los predecesores de Newton. En primer lugar, el uso de prismas para estudiar la refracción de los colores había sido ya practicado numerosas veces. Henry Guerlac atribuye a Descartes el primer estudio sistemático de la proyección del espectro luminoso así como la idea de que la luz no es solamente un auxiliar para la proyección de los colores, sino la fuente misma de ellos (ver 1986). Boyle, por su parte, había realizado varios experimentos con prismas y los había caracterizado como «the usefullest Instrument Men have yet imploy'd about the Contemplation of Colours» (Guerlac 1986; 19). Así que es plausible que el experimento de las Quaestiones haya sido realizado a la luz de los trabajos de Boyle y en el marco de las reflexiones de Descartes, Gassendi y Charleton, 
quienes veían en los colores ya no cualidades propias de los cuerpos sino modificaciones cambiantes de la luz.

Es notable una sentencia que Newton escribe al principio del folio 64: «Note that slowly moved rays are refracted more then swift ones» (1664). Esta afirmación parece ir, sin duda, más allá de los meros fenómenos, pues no existe modo alguno de comprobar la diferencia de velocidades de uno $u$ otro rayo. Esto es suficiente para que Newton no haya dado seguimiento a este tema en posteriores investigaciones. Como hace notar Michael Ben-Chaim, esta clase de formulaciones pertenecerían al tipo de hipótesis que no pertenecen a la ciencia experimental (ver 2001; 410). Esto tiene una relevancia primordial para el análisis que realizaremos posteriormente sobre la naturaleza de los trabajos científicos de Newton en torno a la luz y al color.

En un posterior ensayo llamado «Of Colours» (1665-6), Newton realiza una serie de nuevos experimentos que le permiten manejar los colores de un modo más atinado utilizando, en lugar de pantallas observadas a través de prismas, rayos de luz que atravesaban estos prismas y se refractaban en un iris. Para Ben-Chaim, el sistema práctico de observación que Newton registró en «Of Colours» fue el elemento primordial para lograr el paso de las tesis hipotéticas de las Quaestiones a las conclusiones definitivas a las que llegará en textos posteriores.

\section{La Nueva teoría sobre la luz y el color de Isaac Newton}

El texto que dio a conocer de un modo definitivo las reflexiones de Newton en torno a la luz y el color fue publicado en 
1672 (hay que decir que los textos mencionados hasta este punto nunca fueron publicados por su autor). Newton envió el 6 de febrero una carta dirigida a Henry Oldenburg, Secretario de la Royal Society of London; la carta fue intitulada con el nombre de «New Theory about Light and Colours» 6 . La carta fue leída el día 8 del mismo mes ante los integrantes de la Sociedad y publicada el 19 de febrero de 1672 en los Philosophical Transactions of the Royal Society. La carta está escrita en un estilo anecdótico y, como dice Kuhn (ver 1958; 29), casi autobiográfico ${ }^{7}$. Este carácter estilístico será fundamental para entender la posterior suerte con la que cuenten las ideas aquí expresadas.

En este breve texto, Newton expone lo que supone ser un afortunado descubrimiento mientras observaba el ya ampliamente conocido fenómeno de la refracción de colores a través de un prisma. Newton dice haber oscurecido su cuarto y hecho un hoyo en una ventana; además, se procuró un prisma triangular de vidrio para observar los fenómenos ya mencionados. Nuestro autor nos relata cómo, después de haberse estado «divirtiendo» mientras observaba los intensos colores reflejados, notó un fenómeno que lo sorprendió: «I became surprised to see them in an oblong form; which, according to the received laws of Refraction, I expected should have been circular» $(1672 ; 3076)^{8}$.

Aquí aparece la primera complicación exegética, pues es extraño que Newton se sorprendiera de observar algo que era completamente esperable, a saber, que los rayos que atra-

\footnotetext{
${ }^{6}$ El nombre fue asignado por el mismo Oldenburg (ver Guerlac, 1986).

${ }^{7}$ Es importante notar que el mismo Newton reconoció que, de haber sabido que sería publicada su carta, hubiera introducido algunas anotaciones extra. De hecho, como en muchos otros de sus trabajos, él mismo se mostró inicialmente reacio a su publicación.

${ }^{8}$ El segundo número se refiere a las páginas de Philosophical Transactions.
} 
viesan una superficie inclinada a través de dos medios con índices de refracción diversas se refractarían, es decir, recibirían una cierta deformación. Además, esta refracción debería ser oblonga, ya que el ángulo de incidencia sólo afectaba en un plano (en este caso, en la altura). Sin embargo, Newton parece asumir que, de algún modo, esto contradice a las «leyes de la refracción» que había recibido de la tradición ${ }^{9}$.

Existe otro problema en la interpretación de este pasaje, y es que Newton no da, inicialmente, mayores datos sobre el experimento que genera el fenómeno del que nos habla. Es necesario asumir, por ejemplo, que el hoyo por el cual pasa la luz en la ventana es circular, pues, de lo contrario, Newton no esperaría una refracción con esa figura (ver Sabra 1981; 235); además, debemos suponer que el prisma triangular está colocado con su eje principal (el que va del incentro de una cara triangular al incentro de la otra) paralela al suelo. El ángulo de incidencia y refracción estarán determinados, entonces, por el ángulo que adopten los lados de las caras de los triángulos respecto al piso. De este modo, tiene sentido que la elongación del círculo se diera en la vertical y no en la horizontal de éste.

Sin embargo, continúa sin resolverse el problema de por qué a Newton habría de sorprenderle esta elongación. El lector que, con un poco de conocimientos en materia óptica, se enfrente en primera instancia al texto newtoniano, no podrá sino quedar atónito ya que, en realidad, el problema no pue-

\footnotetext{
${ }^{9}$ Las leyes de la refracción a las que se refiere son, principalmente, la ley de Snell y el principio de Fermat. La primera afirma que el índice de refracción de un medio (n1) por el seno del ángulo de incidencia (q1) es igual al índice de refracción del medio en el que se refracta (n2) por el seno del ángulo de refracción (q2) [n1· q1=n2· q2]. (Para la formulación original, ver Sabra 1981; 11, n.11). La segunda afirma que «el trayecto seguido por la luz al propagarse de un punto a otro es tal que el tiempo empleado en recorrerlo es el mínimo» (Sabra 1981; 136ss).
} 
de ser resuelto sin referencia a otros escritos, pues la carta misma no nos da pistas para entender a Newton. Esta misma confusión fue la que generó la carta entre sus contemporáneos quienes no entendieron, en primera instancia, las reflexiones de Newton y, en algunos casos, ni siquiera sus experimentos ${ }^{10}$. Para entender con mayor facilidad estos textos es necesario acudir a las Lectiones Opticae, escritas entre 1669 y 1671, o a la misma Óptica de 1704. Sin embargo, aunque las Lectiones Opticae fueron escritas antes de la carta a Oldemburg, no fueron publicadas sino hasta 1728. Esto explica por qué, en su momento, la carta de Newton hubiera confundido de tal modo a los científicos.

En la Óptica hay un tratamiento mucho más detallado del mismo experimento relatado en la carta a la Royal Society. Ahí son dadas las especificaciones que antes extrañábamos y se entiende por qué a Newton le sorprendía la elongación de la imagen refractada. Ahí nos dice cómo, al girar el prisma sobre su eje, la imagen refractada se alargaba más o menos; fue entonces cuando decidió fijar el prisma en la posición en la que no hubiera refracción; es decir, en el punto en que los rayos de salida del prisma y los de entrada fueran exactamente el mismo.

If the Prism, whereby so great a Length of the Image was less, the Breadth remaining the same. If the Prism was turned about its Axis that way which made the Rays emerge more obliquely out of the second refracting Surface of the Prism, the Image soon

\footnotetext{
${ }^{10}$ Las principales discusiones en dos de las tres más importantes controversias epistolares que desencadenó la carta de 1672, tienen como punto de partida estas confusiones. Nos referimos a las polémicas con Pardies y Huygens. La tercera polémica, y la más interesante, es la entablada con Robert Hooke (ver Sabra 1981; 236, n.9).
} 
became an Inch or two longer, or more; and if the Prism was turned about the contrary way, so as to make the Rays fall more obliquely on the first refracting Surface, the Image soon became an Inch or two shorter. And therefore in trying this Experiment, I was so curious as I could be in placing the Prism by the abovemention'd Rule exactly in such a Posture, that the Refractions of the Rays at their Emergence out of the Prism might be equal to that at their Incidence on it (1952, libro I, par. 1, prop. 2, teo. 2, 29) ${ }^{11}$.

En efecto, si Newton lograra, mediante la colocación exacta del prisma sobre su propio eje, igualar los rayos de entrada y los rayos de salida del prisma, la imagen refractada debería ser un círculo, al igual que la imagen entrante. Sin embargo, por simple observación de las condiciones del prisma (es decir, por su forma triangular) es evidente que no es posible hacer esto en un solo rayo de luz, pues la cara del prisma en la que entra la luz y la que sale nunca conformarían una refracción perfectamente simétrica. Newton salva este problema considerando en sus cálculos matemáticos únicamente dos rayos del haz de luz que entra por la ventana: el extremo superior y el extremo inferior. Así, coloca el triángulo en la posición exacta para que el ángulo de incidencia del rayo superior sea idéntico al rayo de refracción inferior y, a su vez, que el ángulo de incidencia inferior sea igual al ángulo de refracción del rayo superior. Con esto garantiza que, aunque al revés, el haz de luz que entra en el prisma (considerado en su totalidad) sea idéntico al haz que sale de él (ver Newton 1952; 31-32). Hecha esta precisión, es

\footnotetext{
${ }^{11}$ En las Lectiones Opticae encontramos una proposición semejante: «Since in doing these experiments I supposed the prism's position to be such that rays would be refracted equally at each face of the prism» (Newton 1984, Lecture I, art. 10, 61).
} 
claro que, según las leyes de la óptica que se tenían hasta ese momento, la imagen debería ser un círculo perfecto igual al que entraba en el prisma. Es comprensible ahora que Newton se sorprendiera al ver una figura oblonga y coloreada.

Todo este complicado procedimiento es obviado en la carta de 1672, y simplemente dice que le parece sorprendente encontrar una especie de rectángulo vertical coronado con dos semicírculos en la parte superior e inferior. Ante tal sorpresa, Newton se propone descartar cualquier «irregularidad contingente» que produzca tal efecto: para ello se aboca a cambiar los tipos y tamaños de prismas y los diámetros de las aberturas en la ventana, sin embargo los resultados fueron los mismos (ver Newton 1672; 3076). Finalmente, para eliminar cualquier sospecha de factores contingentes, recurre a un experimento ciertamente ingenioso: coloca un prisma igual al primero (pero en posición contraria) que refracte en sentido opuesto los rayos que atraviesan al primero. Con esto - piensa Newton- «the regular effects of the first Prisme would be destroyed by the second Prisme, but the irregular ones more augmented, by the multiplicity of refractions» $(1672,3076)$. Así, Newton piensa que los factores irregulares o contingentes que introduzcan los prismas se aumentarán, pues una malformación del segundo vidrio no eliminaría otra malformación del primero, sino que ambas se sumarían en una mayor distorsión del espectro. En cambio, los efectos regulares o necesarios de los prismas sí podrían eliminarse mediante la contraposición de cada uno de ellos ${ }^{12}$. El resultado da final-

\footnotetext{
${ }^{12}$ Esta premisa puede ser dudosa, pues no se está completamente cierto de que los efectos regulares se reduzcan siempre negativamente. Sin embargo, para las intenciones de Newton, es válido, ya que lo que interesa mostrar no es que todo efecto regular es reductible mediante la contraposición de los prismas, sino que, si hay un efecto reductible, éste debe ser un efecto regular y no contingente. Dado que la oblicuidad es un efecto reductible,
} 
mente la razón a Newton, pues la proyección de ambos prismas es el de una imagen perfectamente orbicular (incluso más que cuando se proyectaba la luz sin intervención alguna de prisma). Esto implicaba que la refracción oblonga de los rayos formaba parte de los efectos regulares del prisma.

A esta altura es notorio que la descripción anecdótica de Newton es falsa. En primer lugar, porque nosotros, a diferencia de aquéllos a quienes escribía, sabemos que sus investigaciones precedentes le habían llevado poco a poco a estas conclusiones. En segundo lugar, sabemos que el único modo de que fuera posible considerar como sorprendente la elongación del espectro, era que éste apareciera cuando el prisma estuviera colocado en una posición de refracción mínima. Esto implicaba encontrar el ángulo de inclinación preciso para un ángulo determinado del rayo incidente y para una figura particular del prisma; se requería — como vemos en la Óptica y en las Lectiones Opticae - un esfuerzo matemático considerable y una observación precisa que ninguno de sus predecesores (Descartes, Grimaldi, Hooke, Boyle) había notado en experimentos similares ${ }^{13}$. Tampoco es plausible que los eventos descritos por Newton se hayan desarrollado de ese modo ya que el sol, fuente de iluminación en el experimento, se estaría moviendo continuamente y, bajo los criterios de precisión que se necesitaban, no pudo haber sido realizado en un solo día. Resulta sospechoso, entonces, el estilo con que escribe Newton sobre sus descubrimientos

\footnotetext{
Newton puede concluir que es regular.

${ }^{13}$ Como dice Thomas Kuhn: «To destroy the modification theory it was necessary to notice a quantitative discrepancy between the elongation predicted by that theory and the elongation actually observed, and this required an experimenter with a knowledge of the mathematical law governing refraction (not announced until 1637) and with considerable experience in applying the law to optical problems. In 1666 these qualifications were uniquely Newton's» $(1958 ; 32)$.
} 
científicos aunque, como veremos, estos rasgos nos serán de utilidad para reflexionar en torno a las intenciones que tiene nuestro autor al escribir la carta.

\section{El experimentum crucis}

Dentro de la misma carta a la Royal Society, Newton expone lo que él considera el «experimento crucial» de su investigación. Una vez eliminados todos los posibles factores contingentes de refracción, Newton se dispone a encontrar la causa real de la elongación que presenta esta anomalía — en el sentido kuhniano (ver 1958; 32) — dentro de la ciencia óptica de su tiempo.

La primera hipótesis que Newton se plantea supone que, si la luz estuviera compuesta por pequeños cuerpos esféricos, éstos, al golpear una superficie inclinada, serían rebotados con un movimiento de rotación que generaría un desplazamiento en línea curva, al igual que una pelota de tenis cuando es golpeada oblicuamente ${ }^{14}$. Sin embargo —afirma Newton- esto no parece ser así, ya que los rayos parecen no presentar esa curva y, además, la relación entre el diámetro del orificio por donde pasaba la luz y la elongación de la imagen eran proporcionales con la distancia entre el prisma y la imagen de proyección. Esto quiere decir que los rayos no podían generar movimientos curvos, pues la proporción se hubiera perdido ${ }^{15}$. Es importante notar que esta hipótesis

\footnotetext{
${ }^{14}$ El ejemplo es de Newton (ver 1672; 3078).

${ }^{15}$ Se asume aquí que la proporción que se hubiera perdido es una lineal y no geométrica. Es importante notar también que, aunque Newton observa que los rayos no generan una curva, no se siente completamente seguro hasta que analiza la relación proporcional entre distancia y elongación. Esto nos indica la importancia que da Newton a la congruencia
} 
descartada rápidamente por Newton se trata de una posible explicación del fenómeno a partir de principios cartesianos (ver Sabra 1981). En efecto, Descartes intentaba explicar la variación cromática mediante el movimiento de rotación de las partículas del segundo elemento de su sistema natural (ver Guerlac 1986).

Newton procede entonces a realizar su experimentum crucis. Éste consistía en aumentar un segundo prisma al primer experimento y dos cartones con un orificio entre ellos. Así, la luz habría de pasar por la ventana y atravesar el primer cartón a través del orificio; después pasaría por el primer prisma que refractaría el iris alargado, tal como se observó en el primer experimento. El segundo cartón, con su respectivo orificio, serviría para dejar pasar uno, y sólo un color del espectro a través de él. Este rayo separado alcanzaría al segundo prisma que lo refractaría una vez más. Así, el primer prisma podía ser girado para reflejar alternativamente sobre el segundo todos y cada uno de los colores del espectro.

Antes de continuar, es necesario decir que la idea que Newton está tratando de refutar con su experimento es la «teoría de la modificación» del color, que circulaba con cierta aceptación dentro del ambiente científico de la época. Esta teoría sostenía que el color es fruto de una modificación de la luz blanca en el proceso de refracción ${ }^{16}$. En este sentido, el resultado del experimentum fue clave para los intereses de Newton:

matemática sobre los fenómenos.

${ }^{16}$ Steinle (1993a), siguiendo a Nakajima, nos ofrece una síntesis general y sintética de la tesis de la modificación: «Colours are not original properties of light. They are generated in particular interactions with material media by certain modifications which matter causes in light». 
And I saw by the variation of those places, that the light, tending to that end of the Image, towards which the refraction of the first Prisme was made, did in the second Prisme suffer a Refraction considerably greater than the light tending to the other end. And so the true cause of the length of that Image was detected to be no other, then that Light consists of Rays differently refrangible, which, without any respect to a difference in their incidence, were, according to their degrees of refrangibility, transmitted towards divers parts of the wall $(1672 ; 3079)$.

Con esta conclusión, Newton asegura que la refracción no es la causa directa del color al modificar la luz, pues habiendo una segunda refracción, debería haber entonces un nuevo cambio de color.

Newton continúa su texto con una digresión en torno a la construcción de telescopios ${ }^{17} \mathrm{y}$, después, presenta sus conclusiones en trece puntos fundamentales. En resumen, Newton afirma que: (1) los rayos de luz de diversos colores difieren en su grado de «refractabilidad». No son cualificaciones de la luz provocadas por la refracción, sino propiedades originales y «conativas». Es decir, la luz blanca está compuesta de una multiplicidad de rayos de un solo color. (2) Además, cada color está relacionado con un grado de refractabilidad y cada grado de refractabilidad está relacionado con un color específico. (3) Este grado de refractabilidad no es modificable por método alguno conocido (ni refracción ni reflexión). (4) El único modo de lograr la transmutación de éstos es mediante la combinación de varios rayos, pero esta transmutación es llamada así en un sentido poco preciso. (5) Hay, por lo tanto

\footnotetext{
${ }^{17}$ Es importante notar que la investigación de Newton tiene su origen en el intento de reducir la aberración cromática de los telescopios de refracción.
} 
dos tipos de colores: compuestos y primarios. Entre los primarios se encuentran el rojo, amarillo, verde, azul, violeta o púrpura, naranja e índigo (y una indefinida cantidad de gradaciones intermedias ${ }^{18}$ ). (6) Los colores del iris pueden ser, a su vez, formados por dos de los colores adyacentes. (7) El color blanco requiere de la combinación de todos los colores. (8) Esta combinación que produce el blanco debe ser, a su vez, proporcional; si hay uno en mayor grado, el blanco tiende hacia ese color. (9) La refracción de los colores genera que éstos lleguen en un lugar distinto dentro de la retina en el ojo y se genere una diferente percepción de ellos. (10) El arcoíris es sólo una instancia de este fenómeno causado por las gotas de lluvia. (11) Los objetos «tornasol» son un fenómeno explicable por estos principios, pues en diferente posición reflejan distintos rayos. Cuando son iluminados por un rayo puro del espectro pierden esta capacidad. (12) Esto explica también que colocado un recipiente que deje pasar únicamente rayos azules y otro que deja pasar únicamente rayos rojos, se conviertan, una vez sobrepuestos, en opacos ${ }^{19}$. (13) En conclusión, el color de los cuerpos no es otra cosa que una diversa cualificación para reflejar un tipo de luz en lugar de otra. Así, cualquier objeto iluminado por un rayo simple se ve del mismo color del rayo independientemente de cómo se presente habitualmente frente a la luz compuesta (ver 1672; 3081-3085).

Newton llega así a la conclusión de que la luz está compuesta por distintos rayos de distintas coloraciones y que estas cualidades no pueden ser cambiadas. Cada rayo es origi-

\footnotetext{
${ }^{18}$ La inserción de esta aclaración es, evidentemente, muy problemática, pero nos ocuparemos posteriormente de ella.

${ }^{19}$ Se refiere a un experimento realizado por su posterior polemista, Robert Hooke.
} 
nalmente de un color y la blancura es únicamente un efecto derivado de la conjunción de éstos. Esto lleva a Newton a dar vuelta a la hoja de varios cuestionamientos que predominaron en las investigaciones sobre la luz: «whether there be colours in the dark, nor whether they be the qualities of the objects we see» $(1672 ;$ 3085). No queda claro, sin embargo - continúa Newton-, si la luz es un cuerpo o no. No obstante, de no ser un cuerpo, se tendría que afirmar que una cualidad es el substrato de otra, lo cual parece ciertamente absurdo. En efecto, si el color es una cualidad de los rayos de luz, los rayos mismos no podrían ser, a su vez, una cualidad. Esta afirmación pone a Newton, sin afirmarlo, en los linderos del corpuscularismo en la óptica. Esto, como veremos, será fundamental en las posteriores controversias.

\section{Reflexiones en torno a New theory about light and colours}

La carta que acabamos de analizar fue, en definitiva, un texto revolucionario. Los estudios en torno al color y a la luz habían estado históricamente separados; el estudio del color estaba distanciado de la óptica en general (que se había abocado principalmente a la deducción de las leyes de refracción y reflexión) y estaba relegado para el trabajo de los artistas y filósofos especulativos. Desde Euclides se dio esta separación en la teoría del color y no fue sino hasta la modernidad que se reconciliaron esos mundos (ver Guerlac 1986; 14). Esta reconciliación tuvo lugar a partir de la eliminación de la diferencia entre «color real» y «color enfático» que tomó fuerza en el Medioevo y que intentaba asignar al color un carácter inherente a los objetos. El análisis newtoniano fue el 
carpetazo final a esta concepción objetualista. Si algo queda claro con los experimentos de Newton es que los objetos no tienen un color propio, sino una mera cualidad para reflejar primordialmente unos colores sobre otros. Si un objeto es iluminado por un rayo puro, el objeto no puede sino verse de ese color.

Así, cuando Newton asigna a cada color un grado de refractabilidad, reintegra a los colores al mundo del análisis matemático y de la óptica, pues encuentra un modo cuantitativo de describir la escala cromática. No obstante, Newton es consciente de que la interpretación subjetiva de los colores no es, ciertamente, un elemento cuantitativo:

Besides, whoever thought any quality to be a heterogeneous aggregate, such as Light is discovered to be. But, to determine more absolutely, what Light is, after what manner refracted, and by what modes or actions it produceth in our minds the Phantasms of Colours, is not so easie. And I shall not mingle conjectures with certainties $(1672 ; 3085)$.

Esta expresión — que ciertamente nos recuerda al «hipothesis non fingo» de los Principia (ver Newton 2004, libro III, Escolium, 785) - nos indica que Newton no pretende haber descubierto, al menos en este texto, la naturaleza de la luz y el modo en que es percibida por las mentes ${ }^{20}$, únicamente ha perfilado una descripción del comportamiento de la refracción de los rayos de luz. Tenemos, entonces, un primer

${ }^{20}$ Llama la atención la referencia al concepto escolástico de «fantasma» como la imagen mental del color. Aquí, como en la discusión sobre el carácter cualitativo o sustancial de la luz se notan rasgos de aristotelismo en Newton. Esto no es algo completamente extraño en su pensamiento, en el De gravitatione et aequipondio fluidorum encontramos también un análisis del espacio en términos de categorías aristotélicas: ver Newton 2006, 41, folio 131. 
problema heurístico en nuestro texto: por una parte, Newton parece no dar un paso más allá de los fenómenos, pero, como habíamos visto antes, parece aproximarse bastante a una posición corpuscularista de la luz, lo cual parecería contradecir su principio metodológico.

La redacción de la carta encierra un segundo punto crucial de análisis. En efecto, observamos que Newton de algún modo miente acerca del desarrollo biográfico en que fue realizada su investigación y lo relata como resultado de una sutileza en la observación. Esto, como ya vimos, es falso, y parece ser fruto de un arduo trabajo de investigación. Parece que Newton, más que encontrarse con un fenómeno anómalo de la naturaleza, sentó a la luz en «el banquillo de los acusados», por decirlo en un tono kantiano. Sin embargo, la pregunta de por qué siguió este método tan poco ortodoxo a la hora de presentar sus conclusiones continúa abierta, pero nos puede indicar líneas interesantes para descifrar la intención epistemológica que yace detrás del texto ${ }^{21}$.

En tercer lugar, Newton asume dejar a un lado la versión modificacionalista de la teoría del color. Esto, como veremos posteriormente, no será del todo aceptado por los científicos de su tiempo y generará una de las más interesantes controversias en las que haya estado envuelto Newton.

\footnotetext{
${ }^{21}$ Ciertamente, esto puede ser fruto de que el texto fue publicado a través de una revista, lo cual, como nos explica Kuhn (1958), era algo verdaderamente novedoso; las grandes aportaciones de la ciencia hasta ese momento habían sido presentadas a través de libros completos. Esto pudo haber restado seriedad al discurso newtoniano, sin embargo, no deja de ser curioso que, tratándose de un hombre tan obsesivo y cuidadoso con sus publicaciones, haya tratado un tema de tal profundidad y relevancia en este modo. No olvidemos que los Principia se publicaron gracias únicamente a la insistencia de su amigo Halley, pues de lo contrario, su personalidad perfeccionista no le hubiera permitido publicar un trabajo, para él, tan incompleto (ver Keynes 1963; 464).
} 
Por último, aparece el complicado problema de identificar cuántos y cuáles son los colores del espectro cromático. Newton parece haber escogido siete colores dentro de una «infinidad de tonos intermedios».

Me interesa hacer énfasis en estos dos últimos puntos, a saber, la elección de las divisiones cromáticas y la polémica con el modificacionalismo, para desprender de ahí ciertas reflexiones epistemológicas respecto a la investigación newtoniana.

\section{Divisiones de la escala cromática}

Es claro que Newton, cuando observa el iris proyectado por los prismas no encontró divisiones precisas entre uno y otro color. Él mismo parece ser consciente de esto y lo menciona tanto en la carta de 1672 como en la Óptica (ver 1952, libro I, par. II, prop. II, teo. II, 122). Sin embargo, Newton no parece titubear al dividir el espectro en siete colores básicos o primarios.

No obstante, en sus investigaciones precedentes a 1672, no cuenta con tal seguridad a la hora de dividir el iris. En efecto, en sus primera Lección de las Lectiones Opticae, Newton parece asignarle cinco colores: rojo, amarillo, verde, azul y morado. Sin embargo, como bien nota David Topper (1990) en la decimoprimera Lección, Newton pasa a hacer la división de siete colores que serán mencionados en la carta de 1672 y en la Óptica (aumentando el índigo y el naranja).

Muchas especulaciones se han realizado en torno al porqué de este cambio. Bierson, por ejemplo, argumenta que Newton tomó la enumeración de los colores de la paleta de de un pintor, argumentando que el índigo no es precisamen- 
te un color que se distinga con facilidad ${ }^{22}$. Armstrong, por su parte, parece decir que Newton vio siete colores porque realmente hay siete colores para ver, lo cual sólo traslada el problema a pensar por qué vio originalmente cinco. Penelope Gouk, por su parte, explica este cambio como parte de una supuesta analogía entre los colores y la música, identificando la escala tonal con el espectro cromático. Topper, por su parte, pensará que la reconsideración de Newton es un factor estético en la división de la gama. Es decir, que una división en siete partes ofrecía a Newton una mayor simetría en el espectro que una división en cinco, ya que los registros azules y morados ocupaban una mayor parte del iris que los colores rojos y amarillos ${ }^{23}$. La analogía musical, indica Topper, puede ser posterior a esta división, cuando Newton se da cuenta que la separación en siete colores es congruente con las notas y que el espacio entre sí guarda también relación con las leyes de la armonía.

De cualquier modo, siendo cualquiera de estas hipótesis la verdadera, es cierto que la división en siete tonos tiene un origen extra-fenoménico pues, en efecto, la dificultad para atribuir puntos claros de división obliga a Newton a recurrir a otros criterios que van más allá de la mera observación ${ }^{24}$. No quiere decir esto que inventara fenómenos, sino que lo que de hecho veía, era interpretado bajo un marco teórico mucho más amplio que el de la ciencia experimental (sea el de la teoría musical, el de la matemática o el de las proporciones estéticas).

\footnotetext{
22 Para una más amplia descripción de esta discusión, ver Topper 1990.

${ }^{23}$ Nótese la complicación de esta hipótesis: el espectro dividido en siete colores tampoco es, en lo absoluto, simétrico.

${ }^{24}$ Newton parece protegerse frente a esta crítica al aclarar en las Lectiones Opticae que invitaba a un observador imparcial a colocar las divisiones de los colores en el espectro. Sin embargo, no dice si a este observador neutro se le pedía que pusiera cinco o siete divisiones o si se dejaba a su elección (ver Topper 1990; 278).
} 


\section{La discusión con Hooke}

El texto de Newton, una vez presentado a la Royal Society, despertó gran interés y polémica. Una respuesta crítica llegó apenas una semana después por parte del científico y miembro de la Royal Society, Mr. Robert Hooke.

La respuesta de Hooke es breve y concisa. Sorprendentemente, sus críticas tienen poco o nada que ver con la elaboración de los experimentos y la recolección de los fenómenos. Lo interesante en la respuesta de Hooke es que su crítica se basa en desplazar toda la teoría newtoniana a la razón de una mera hipótesis. Esto para Newton era traducible a un derrumbamiento de su teoría pues, para él, las hipótesis no podían permanecer en el discurso científico sino como puros medios para llegar a una conclusión, pero no como las conclusiones mismas (ver Sabra 1981; 231).

Hooke (1958; 111 [11]) va a aceptar que los experimentos llegan a las conclusiones correctas, pero que de éstos no se sigue la conclusión principal de Newton, a saber, que la luz es un compuesto de rayos genuinamente cualificados con un color: «But why there is a necessity, that all those motions, or whatever else it be that makes colours, should be originally in the simple rays of light». Hooke, asegura, además, que no hay razón para preferir el sistema newtoniano siendo que hay muchas otras posibilidades de salvar los fenómenos: «for the same phenomenon will be solved by my hipothesis». Esto destruiría por completo las tesis newtonianas que pretendían mostrarse como indiscutibles. La última crítica fundamental de Hooke es acusar a Newton de haber afirmado que la luz era corpórea sin tener ningún indicio real. Nuevamente, critica la poca relación entre las hipótesis generales y los fenómenos que recolecta Newton. 
En la respuesta a las críticas, Newton se ocupará de deslindarse de la hipótesis de la luz como un cuerpo. Efectivamente, observábamos que el análisis de Newton deja abierta la posibilidad (y casi la afirma), aunque no concluye a favor de ella. Newton se da cuenta de que si se niega su teoría de la composición de la luz o se reduce a una mera hipótesis, su aportación sería nula: «There would be little or not difference between Us» (1958; 122 [5090]). Newton argumenta con razón que es absurdo que a una sustancia homogénea (como supone Hooke que es la luz) aplicándole la misma modificación (la refracción dispersante) genere distintos efectos (a la luz blanca, la separación de los colores; a la luz simple, ninguno), y que distintas modificaciones (la refracción conjuntante) generen un efecto homogéneo (la luz blanca).

Con este argumento, Newton parece atinar al problema de la teoría modificacionalista de Hooke, sin embargo, como dice Sabra (1981), no es absolutamente concluyente mediante los experimentos que la luz sea un heterogéneo de colores; a lo mucho, se puede decir que cada color posee su propio y constante grado de refractabilidad. En efecto, del hecho de que no haya una modificación de los rayos de luz en la segunda proyección del experimentum crucis, no se sigue que la efectiva y real causa de la primera modificación no fuera la primera refracción. Ciertamente, el experimento da unas ciertas pautas de probabilidad que colocan a la hipótesis de Newton en una situación muy cómoda, pero en lo absoluto definitiva.

Este es el segundo ejemplo en donde vemos que Newton rebasa de algún modo los fenómenos para establecer una tesis que sostiene como verdadera y comprobable. Primero fue la división del espectro cromático; en segundo lugar, la afirmación categórica de la validez de la teoría de la composición de los colores en un haz blanco. 


\section{Conclusiones}

Hemos hecho un análisis del desarrollo de la teoría newtoniana de la luz y el color a lo largo de buena parte de su trabajo científico y filosófico. Encontramos un paulatino desarrollo de las tesis que Newton presentará como definitivas en 1672 ante la Royal Society. Vimos también cómo, en los primeros textos de juventud, Newton descarta ciertas hipótesis por ir más allá de los fenómenos (en concreto, la teoría de los giros en las partículas de luz). Sin embargo, hemos encontrado también que en su doctrina definitiva hay elementos extra-fenoménicos y no experimentables que ponen en jaque a las propias demostraciones newtonianas. Esto se ve acentuado cuando Newton se esfuerza en negar que estas razones sean hipotéticas en su correspondencia con Hooke. Cabe preguntarnos, entonces, ¿qué diferencia existe entre las hipótesis que el mismo Newton desecha en sus textos de juventud y estas conclusiones extrafenoménicas que defiende como científicas y verdaderas? Desde luego, la defensa que hace de las segundas puede tener simplemente como causa el orgullo y soberbia de Newton ${ }^{25}$, pero también es difícil pensar que nuestro autor, por su capacidad y personalidad, hubiera publicado una tesis de la cual no se encontrara plenamente convencido.

Encontramos, pues, en el planteamiento de Newton, distintas afirmaciones en las que se ve rebasado el ámbito de los fenómenos para establecer conclusiones teóricas de modo conclusivo: a) La afirmación inválida de que la teoría modificacionalista se encontraba absolutamente refutada por los fenómenos extraídos de su investigación; b) la división en

\footnotetext{
${ }^{25}$ Como dice, Mamiani, la controversia con Hooke perduró hasta la muerte de éste por causas más personales que científicas (ver Mamiani 2000; 151).
} 
siete colores de la escala cromática que, como hemos visto, no puede ser explicada por los fenómenos; y c) sus afirmaciones en torno a la naturaleza corpórea de la luz. Esto, por otra parte, contrasta con pautas metodológicas que Newton sigue $y$, en ocasiones, hace explícitas continuamente: a) su desdén por las hipótesis, reflejado principalmente en textos como los Principia y la Óptica y, como vimos, en su discusión con Hooke; y b) su empeño por mantenerse al margen de cuestiones metafísicas en los textos publicados.

Una lectura cientificista de Newton no puede, desde mi perspectiva, reconciliar estas aristas que presenta su estudio. Tendría que asumir, como hacen Mach o Hooke, que sus teorías son inválidas por rebasar los fenómenos, o que simplemente los textos de juventud poseen poca importancia para entender lo que verdaderamente quería hacer Newton con su ciencia. La consecuencia de esto sería perder los marcos conceptuales que nos posibilitarían dar sentido a la ciencia newtoniana tal. Las investigaciones de óptica ponen en claro que la mera referencia a los fenómenos no cumple satisfactoriamente con las expectativas epistemológicas de Newton. Es cierto que el apego de Newton a los fenómenos es su condición primordial para el desarrollo científico, pero en casi todos sus ámbitos de investigación observamos una subsunción de los fenómenos a un marco de interpretación mucho mayor. Efectivamente, las hipótesis no tienen lugar en la filosofía experimental ${ }^{26}$, pero no toda la filosofía es experimental para Newton ${ }^{27}$.

\footnotetext{
${ }_{26}$ «Pues lo que no se deduce de los fenómenos, ha de ser llamado Hipótesis; y las hipótesis, bien metafísicas, bien físicas, o de calidades ocultas, o mecánicas, no tienen lugar dentro de la Filosofía experimental» (Newton 2004, libro III, Escolio, 785).

${ }^{27}$ Una clara aportación a esta idea es el artículo de ya citado de Michael Ben-Chaim (2001).
} 
Nuestro autor pretende dejar bien claro en cada uno de sus textos la intención que tiene al escribirlos; en mayor o menor medida, logra atenerse a ello. Esto podría justificar, quizá, la extraña redacción de su carta a la Royal Society donde intenta, ciertamente, dejar de lado cualquier resabio de una idea preconcebida ante la observación de los fenómenos. De algún modo, intenta retratar a un ideal de científico que se «topa», literalmente, con los fenómenos y se atiene exclusivamente a ellos. Pero el hecho de que Newton tome esta perspectiva en sus tratados científicos, no implica que no posea un marco teórico y metafísico bajo el cual entiende y asume esos fenómenos. Es por esta razón que quien se acerca a la obra de Newton puede encontrarse un autor tan variado y disímil que parece ser otro en cada uno de sus textos. Su sistema puede reconstruirse en la suma de toda su obra, pero en cada una de sus partes sólo se encuentran algunos dejos o referencias a otras partes del sistema. Si se leyeran únicamente los Principia sin los Escolios o la Óptica sin las Cuestiones (que, como ya dijimos fueron agregadas por sugerencias de sus editores), no se sospecharía en lo absoluto de la existencia del gran filósofo natural que está detrás de ellos.

¿Qué papel juega entonces el desarrollo científico de Newton en el tema de la óptica y el color? Desde luego, los textos mostrados pretenden ser de carácter científico y experimental. Sin embargo, no dejan por eso de estar enmarcados en una filosofía natural mucho más extensa y profunda. Si se analizan estos textos con una mirada puramente cientificista como, extrañamente para su época, hace Hooke, Newton ciertamente parece ir más allá de los meros fenómenos. En ese sentido, sus afirmaciones generales sobre la luz parecen ser extra-experimentables y, desde una perspectiva cientificista, meras hipótesis. Sin embargo, si se atiende a la filoso- 
fía natural de Newton, estas hipótesis parecen adquirir un sentido coherente y correcto. No sólo eso, parece claro que Newton mismo entiende a la ciencia enmarcada dentro de este marco metafísico ${ }^{28}$, aunque no lo exprese de ese modo en todos sus textos publicados ${ }^{29}$.

Efectivamente, del experimentum crucis no parece seguirse que la luz blanca esté conformada por rayos simples cuyas cualidades son los colores; pero, ¿con qué experimento se podría comprobar la simplicidad de algo? Sin embargo, es cierto también que las conclusiones de Newton son las más plausibles y acertadas, si bien no provienen exclusivamente de los fenómenos, los explican con abundante claridad ${ }^{30}$.

En este sentido, quizá el error de Newton no haya sido mezclar distintos campos del saber —en este caso, ciencia y filosofía - sino mantener la pretensión de preservarlos absolutamente intactos en cada uno de sus textos.

\footnotetext{
${ }^{28}$ Atiéndase, por ejemplo, el inicio y fin de los Principia que, como ya hemos dicho, funcionan como una especie de "paréntesis metafísico» para entender los principios científicos que expone ahí.

${ }^{29}$ Se podría pensar, como hace Koyré, que es preferible hacer una lectura cientificista de Newton dado que todos aquellos textos donde aparecen estas reflexiones metafísicas nunca los publicó, mientras que los textos primordialmente científicos sí fueron publicados (ver $1979 ; 151)$. Esta objeción es inválida pues, siguiendo ese principio, habría que decir que ni siquiera el contenido de los Principia hubiera sido valorado por Newton dado que tampoco estaba en sus planes publicarlo (y no lo hubiera hecho sin la insistencia de su compañero Halley). Esto nos indica que, probablemente, la falta de circulación de sus textos metafísicos se deba más a la personalidad de Newton que a su valoración sobre ellos. Como nos cuenta Keynes: «He parted with and published nothing except under the extreme pressure of friends» (1963; 311-312). Recordemos que el mismo Newton se siente sorprendido de que su carta sobre la luz y el color fuera publicada y admite que, de haberlo sabido, hubiese sido más cauteloso para escribirla (ver Kuhn 1958; 29).

${ }^{30}$ El mismo caso se presenta, como ya decíamos, en el concepto de «espacio absoluto» en los Principia o, incluso, en la noción de «rayo de luz» de la Óptica (ver Steinle 1993b; 570).
} 


\section{BIBLIOGRAFÍA}

Ben-Chaim, Michael. (2001). «The discovery of natural goods: Newton's vocation as an experimental philosopher». British Journal for the History of Science 34; 395-416.

Benítez, Laura. (2003). «La nueva ontología: tras la filosofía natural de Newton-Clarke». En: Hurtado. G., Nudler, O. (eds). El mobiliario del mundo. Ensayos de ontología y metafísica. México: UNAM.

Ducheyne, Steffen. (2001). «Isaac Newton on Space and Time: Metaphysician or Not?». Philosophica 1 (67); 77-144.

Escohotado, Antonio. (1997). «Estudio Preliminar». En: Newton, Isaac. Principios matemáticos de la Filosofía natural. Madrid: Tecnos.

Guerlac, Henry. (1986). «Can there be colors in the dark? Physical color theory before Newton». Journal of history of ideas 1 (47); 3-11.

Hooke, Robert (1958). «Hooke's critique of Newton Theory». En: Cohen, B. (ed). Isaac Newton's Papers and Letters on Natural Philosophy. Cambridge, MA: Harvard University Press.

Keynes, J. Maynard. (1963). Essays in Biography. New York: Horizon Press.

Koyré, Alexandre. (1979). Del mundo cerrado al universo infinito. México: Siglo Veintiuno.

Kuhn, Thomas. (1958). «Nota introductoria» a «Newton's Optical Papers». En: Cohen, B. (ed). Isaac Newton's Papers and Letters on Natural Philosophy. Cambridge, MA: Harvard University Press.

Mach, Ernst. (1960). The Science of Mechanics. Illinois: Open Court. 
Mamiani, Maurizio. (2000). «The structure of a Scientific Controversy». En: Machamer P., [et. al]. (ed). Scientific Controversies. Oxford: Oxford University Press.

Marquina, José. (2006). La tradición de Investigación Newtoniana. México: UAM-I.

McGuire, J.E. (1980). «Space, Geometrical Objects and Infinity». Nature mathematized 1; 69-112.

Newton, Isaac. (2006). De gravitatione et aequipondio fluidorum. En: Benítez, L., Robles, J.A. (eds). De Newton y los newtonianos entre Descartes y Berkeley. Buenos Aires: Universidad Nacional de Quilmes.

Newton, Isaac. (2004). Principios matemáticos de la Filosofía natural. Madrid: Alianza.

Newton, Isaac. (1984). Lectiones Opticae. En: Shapiro, A. (ed). The optical papers of Isaac Newton. Cambridge, UK: Cambridge University Press.

Newton, Isaac. (1958). «Newton's answer to Hooke». En: Cohen, B. (ed). Isaac Newton's Papers and Letters on Natural Philosophy. Cambridge, MA: Harvard University Press.

Newton, Isaac. (1952). Opticks, or a Treatise of the reflections, refractions, inflections $\mathcal{E}$ colours of light. New York: Dover. Newton, Isaac. (1672). «A Letter of Mr. Isaac Newton, Professor of the Mathematics in the University of Cambridge, containing his New Theory about Light and Colors». En Philosophical Transactions of the Royal Society 6; 3075-3087. Newton, Isaac. (1665-6). «Of Colours». Additional Ms. 3975. Cambridge, UK: Cambridge University Library. Versión digital a cargo de Newton Project, http:/ / www.newtonproject.sussex.ac.uk/view/texts/ normalized /NATP00004.

Newton, Isaac. (1664). «Quaestiones quaedam philosophiae». En: Westfall, R. (ed). Newton Papers of Indiana University. Bloomington: Indiana University Library. 
Sabra, A. I. (1981). Theories of light From Descartes to Newton. Cambridge: Cambridge University Press.

Steinle, Friederich. (1993a). "Newton rejection of the modification theory of colour». En: Hegel and newtionanism. Dordrecht: Kluwer Academic.

Steinle, Friederich. (1993b). «Newton's colour theory and perception». En: Hegel and newtionanism. Dordrecht: Kluwer Academic.

Strong, E. W. (1952). «Newton and God». Journal of the History of Ideas 2 (13); 147-167.

Topper, David. (1990). «Newton and the number of colours in the spectrum». Studies in History and Philosophy of Science 2 (21); 269-279.

Toulmin, Stephen. (1959a). «Criticism in the History of Science: Newton on Absolute Space, Time and Motion, II». The Philosophical Review 2 (68); 1-29.

Toulmin, Stephen. (1959b). «Criticism in the History of Science: Newton on Absolute Space, Time and Motion, I». The Philosophical Review 1 (68); 1-29. 


\section{RESUMEN}

El presente artículo pretende hacer, en primer lugar, un análisis de los primeros textos donde Isaac Newton presenta sus experimentos y reflexiones en torno a su teoría de la luz y el color. Mostraré que en estos textos hay problemas hermenéuticos que no pueden ser resueltos sin la consideración de principios extra-fenoménicos en el pensamiento newtoniano. Para ello, haré referencia principalmente a la carta de 1672 dirigida a la Royal Society intitulada «New Theory about Light and Colours». A partir del análisis de ese texto, tomaré en consideración el problema de la división que hace Newton en el espectro de colores y la controversia con la teoría modificacionalista encarnada en la figura de Robert Hooke. Mis conclusiones apuntan a la consideración de una lectura no meramente funcionalista de los principios extra-fenoménicos en el pensamiento newtoniano.

Palabras clave: Newton; color; luz; óptica; Hooke.

\section{ABSTRACT}

The aim of this paper is to analyze the earliest texts where Isaac Newton presents his experiments and reflections on his theory of light and color. I will show that there are hermeneutic problems in these texts that cannot be solved without the consideration of extra-phenomenical principles in Newtonian thought. With this purpose, I will mainly refer to the letter «New Theory about Light and Colours» addressed to the Royal Society in 1672; from this analysis I will take into consideration the problem of Newton's division of the color spectrum and the controversy about the modificationist 
theory, mainly represented by Robert Hooke. My conclusions point toward a consideration of a non-functionalistic lecture of extra-phenomenical principles in Newtonian thought.

Key words: Newton; color; light; optics; Hooke. 